That the laser has had an enormous impact on modern spectroscopy is history. However, this impact was long sequestered in academic pursuits, where a myriad of "new" light scattering phenomena were observed. Most of these were previously unobserved light scattering effects due to second- and thirdorder terms in molecular polarizability. Toward the end of the last decade, the field of laser spectroscopy reached a maturity, and numerous laser spectroscopic techniques began finding practical application.

In this issue of Optical Engineering there are two representative papers in the category of laser spectroscopy. The first paper, "Laser-excited atomic fluorescence techniques for temperature measurements in flames: a summary," by Zizak, Omenetto, and Winefordner, attests to the maturity of laser spectroscopic investigation of flames by espousing and fulfilling the need for a succinct yet comprehensive summary of laser-excited atomic fluorescence methods for absolute measurements of temperature in flames. In the second paper, "Photothermal refraction for scanning laser microscopy," Burgi et al. have taken the effect of thermal lensing, which had become the bane of investigators of nonlinear optical phenomena, and used it to their advantage.
Guest Editorial

\section{Laser Spectroscopy}

Fred P. Milanovich

Lawrence Livermore National Laboratory P.O. Box 5507, L-524

Livermore, California 94550

Stanley M. Klainer

ST\&E Technical Services, Inc. 20 Belinda Court

San Ramon, California 94583 